\title{
On Collineation Groups of Finite Projective Spaces
}

\author{
David Perin
}

\section{Introduction}

Let $V$ be a vector space of finite dimension $n$ over a finite field $G F(q)$. Let $L_{k}(V)$ denote the set of $k$-dimensional subspaces of $V$. Several authors have studied groups acting on $L_{k}(V)$ for various $k$. Wagner [9] considered groups which act doubly transitively on $L_{1}(V)$. Recently Kantor [6] has shown that most groups which act transitively on $L_{2}(V)$ also act doubly transitively on $L_{1}(V)$. This paper considers groups which act transitively on $L_{k}(V)$ for $3 \leqq k \leqq n-3$. The main result is the following theorem.

Theorem 1. Suppose $F \leqq \Gamma L(V)$ acts transitively on $L_{k}(V)$ for $3 \leqq k \leqq n-3$. Then $F \geqq S L(V)$ with the possible exception of $q=2$ and $n$ even.

$\Gamma L(V)$ is the group of all nonsingular semilinear transformations of $V$. The assumption $k \leqq n-3$ is necessary so that elements of $L_{k}(V)$ are not dual to elements of $L_{1}(V)$ or $L_{2}(V)$. If $n=4$, then $\operatorname{Sp}(V)$ is transitive on $L_{3}(V)$ but does not contain $S L(V)$. No counterexamples to Theorem 1 are known to the author in the case $q=2$ and $n$ even, but the proof given here breaks down in this case. The proof consists in showing that $G$ contains a transvection. Then well-known results concerning groups generated by transvections imply that $F$ contains $S L(V)$. A number theoretic result of Birkhoff and Vandiver plays an important role in the proof. Also, the above result of Kantor is used to shorten the original proof and to avoid some difficulty in characteristic 2 .

\section{Previous Results}

In this section we list several results which will be used in the proof of Theorem 1 .

1.1. If $G \leqq \Gamma L(V)$ acts transitively on $L_{k}(V)$ for $k \leqq[n / 2]$, then $G$ acts transitively on $L_{j}(V)$ for $j \leqq k$.

This is an unpublished result of McLaughlin. A sketch of the proof follows. Let $U_{k}$ be a fixed element of $L_{k}(V)$. Let $\Gamma_{k}$ be the stabilizer in 
$\Gamma L(V)$ of $U_{k}$. Then since $k \leqq[n / 2], \Gamma_{k}$ acts on $L_{j}(V)$ as a permutation group with $j+1$ orbits. An orbit consists of all $U \in L_{j}(V)$ such that $U \cap U_{k}$ has dimension $i$. The possible values of $i$ are $0,1, \ldots, j$.

Let $\mu_{j}$ be the permutation character afforded by the action of $\Gamma L(V)$ on $L_{j}(V)$. From the above remarks it follows that $\left(\mu_{i}, \mu_{j}\right)=i+1$ for $i \leqq j \leqq k$. Thus, for example $\mu_{1}=1+\theta_{1}$ and $\mu_{2}=1+\phi_{1}+\phi_{2}$ where $\theta_{1}, \phi_{1}$, $\phi_{2}$ are nonprincipal irreducible characters of $\Gamma L(V)$. Since $\left(\mu_{1}, \mu_{2}\right)=2=$ $1+\left(\theta_{1}, \phi_{1}\right)+\left(\theta_{1}, \phi_{2}\right), \theta_{1}$ must equal $\phi_{1}$ or $\phi_{2}$, i.e., we may write $\mu_{2}=$ $1+\theta_{1}+\theta_{2}$. In a similar fashion it follows that there are distinct nonprincipal irreducible characters $\theta_{1}, \theta_{2}, \ldots, \theta_{k}$ of $\Gamma L(V)$ such that $\mu_{j}=$ $1+\theta_{1}+\cdots+\theta_{j}$.

Now suppose $G \leqq \Gamma L(V)$ acts transitively on $L_{k}(V)$. Then $1=$ $\left(\mu_{k} \mid G, 1\right)=1+\left(\theta_{1} \mid G, 1\right)+\cdots+\left(\theta_{k} \mid G, 1\right)$. Hence $\left(\theta_{1} \mid G, 1\right)=\cdots=\left(\theta_{k} \mid G, 1\right)=0$, and so $\left(\mu_{j} \mid G, 1\right)=1+\left(\theta_{1} \mid G, 1\right)+\cdots+\left(\theta_{j} \mid G, 1\right)=1$. Thus, $G$ acts transitively on $L_{j}(V)$.

Other results of a similar nature can be proved using the same type of argument. For example, if $G$ acts on $L_{k}(V)$ as a rank $k+1$ permutation group, then $G$ acts on $L_{j}(V)$ as a rank $j+1$ permutation group if $1 \leqq j \leqq k \leqq[n / 2]$.

1.2. Suppose $G \leqq \Gamma L(V)$ contains a transvection with center $P$ for each point $P \in L_{1}(V)$. If $V$ has dimension greater than or equal to 3 , then either $G \geqq S L(V)$ or $\operatorname{Sp}(V) \leqq G \leqq N_{\Gamma L(V)}(\operatorname{Sp}(V))$ [7]. In particular if $V$ has dimension greater than 4 and $G$ acts transitively on $L_{3}(V)$, then $G$ contains $S L(V)$.

Let $V$ be an abelian group and $G$ a group which acts on $V$. Then a derivation $\theta: G \rightarrow V$ is a function which satisfies the equation $\theta(x y)=$ $\theta(x) y+\theta(y)$. If $v \in V$, then the function $\theta$ defined by $\theta(x)=v-v x$ is a derivation. Such derivations are called inner derivations. The set of derivations forms an additive abelian group $Z$ under the operation of pointwise addition. The set of inner derivations forms a subgroup $B$. The group $H=Z / B$ is called the group of outer derivations of $G$ into $V$. If $V$ is a vector space over a field $F$, then $Z, B$, and $H$ are also vector spaces over $F$. The following well-known results concerning derivations will be used in the proof of Theorem 1.

1.3. If $V$ is a vector space over the field $F$ and $G$ is a group of linear transformations on $V$ which contains a nonidentity scalar transformation, then $H=0$. In particular for $q$ odd, all derivations of $S L_{2}(q)$ on its standard module, the two dimensional vector space over $G F(q)$, are inner derivations.

1.4. If $q=2^{m}>2, G=S L_{2}(q)$ and $V$ is the standard module of $G$, then $H$ has dimension one over $G F(q)$. Moreover, each coset of $B$ in $Z$ 
contains a representative $\theta_{b}$ such that

$$
\theta_{b}\left|\begin{array}{ll}
1 & 0 \\
a & 1
\end{array}\right|=(b \sqrt{a}, 0)
$$

A divisor $t$ of $q^{n}-1$ is $q$-primitive if $t>1$ and $\left(t, q^{i}-1\right)=1$ for $i=$ $1, \ldots, n-1$. The following results are from [4] although many of the results were known at the turn of the century. For example, see $[1,2]$.

1.5. $q^{n}-1$ has a $q$-primitive divisor except in the following cases: $n=1$ and $q=2 ; n=2$ and $q+1=2^{i} ; n=6$ and $q=2$.

1.6. Suppose $t$ is a q-primitive prime divisor of $q^{n}-1$. Let $T$ be a Sylow t-subgroup of $G=G L(V)$ where $V$ is an $n$ dimensional vector space over the field $G F(q)$. Then $T$ is cyclic and for $1 \neq X \leqq T, C_{G}(X)=C_{G}(T)$ is isomorphic to the multiplicative group of $G F\left(q^{n}\right) . C_{G}(T)$ acts regularly on the nonzero vectors of $V$ and is usually called a Singer subgroup of $G$. In addition $N_{G}(X)=N_{G}(T)$ is isomorphic to $G F\left(q^{n}\right)^{*}$ extended by the subgroup of Aut $\left(G F\left(q^{n}\right)\right)$ which fixes $G F(q)$ elementwise [4]. In particular $N_{G}(X)$ is a metacyclic group of order $n\left(q^{n}-1\right)$. If $H \leqq G$ and $K$ is a normal subgroup of $H$ whose order is divisible by $t$, then the Frattini argument and the above results imply that $H / K$ is a metacyclic group of order dividing $n\left(q^{n}-1\right)$.

1.7. Piper [8]. Let $V$ be a vector space of finite dimension greater than or equal to 3 over a finite field $G F(q)$. If $G \leqq \Gamma L(V)$ acts transitively on $L_{1}(V)$ and contains a dilatation (i.e., a homology in $P \Gamma L(V)$ ), then $G$ contains $S L(V)$.

1.8. Kantor [6]. If $G \leqq \Gamma L(V)$ is transitive on $L_{2}(V)$ but not doubly transitive on $L_{1}(V)$, then $n \leqq 3$ or $n=5, q=2$, and $|G|=31 \cdot 5$.

1.9. Wagner [9]. Suppose $G \leqq \Gamma L(V)$ is 2-transitive on $L_{1}(V)$. Let $1 \neq N$ be a normal subgroup of $G$, and let $U$ be a three dimensional subspace of $V$. Then,

(1) $N$ is doubly transitive on $L_{1}(V)$.

(2) The action of $G_{U}$ on $U$ contains $S L(U)$. Hence, the action of $G_{W}$ on a two dimensional subspace $W$ of $U$ contains $G L(W)$.

(3) If $n \leqq 5$, then $G$ contains $S L(V)$ or else $n=4, q=2$, and $G \simeq A_{7}$.

\section{Proof of Theorem 1}

By 1.1 and duality it suffices to prove Theorem 1 in the case $k=3$. So suppose $V$ is a vector space of finite dimension $n \geqq 6$ over $G F(q)$, and $F \leqq \Gamma L(V)$ is transitive on $L_{3}(V)$ but does not contain $S L(V)$. We show that $q=2$ and $n$ is even. Let $G=F \cap S L(V)$. $G$ acts nontrivially on $L_{1}(V)$ 
since $\left|L_{3}(V)\right|$ divides $|F|$ but not $(q-1)(\Gamma L(V): S L(V))$. Also, $G \neq S L(V)$ by assumption.

$F$ is transitive on $L_{2}(V)$ by 1.1 . Kantor's result implies that $F$ is doubly transitive on $L_{1}(V)$. Thus, $G$ is also doubly transitive on $L_{1}(V)$ by 1.9 .

Let $U$ be a two dimensional subspace of $V$. Let $K$ denote the subgroup of $G_{U}$ which acts on $V / U$ as the identity. Let $L$ be the subgroup of $G_{U}$ which acts on $U$ as the identity. Finally set $J=K \cap L$. Then, $K$, $L$, and $J$ are normal subgroups of $G_{U}$ which can be represented by matrices of the following forms respectively:

$$
\left|\begin{array}{ll}
A & 0 \\
\theta & I
\end{array}\right|, \quad\left|\begin{array}{ll}
I & 0 \\
\theta & B
\end{array}\right|, \quad\left|\begin{array}{ll}
I & 0 \\
\theta & I
\end{array}\right|,
$$

where $A \in G L_{2}(q)$ and $B \in G L_{n-2}(q)$. For $X \leqq G_{U}$ let $\bar{X} \simeq X L / L$ and $\tilde{X} \simeq X K / K$ denote the linear groups induced by the action of $X$ on $U$ and $V / U$ respectively. The above argument and $1.9 \mathrm{imply}$ that $\bar{G}_{U}=$ $G L(U) \simeq G L_{2}(q)$.

$F$ has order divisible by

$$
\left|L_{3}(V)\right|=\left(q^{n}-1\right)\left(q^{n-1}-1\right)\left(q^{n-2}-1\right) /(q-1)\left(q^{2}-1\right)\left(q^{3}-1\right) .
$$

Since $n \geqq 6,1.5$ implies that $|F|$ is divisible by a $q$-primitive prime divisor $t$ of $q^{n-2}-1$ except for $q=2$ and $n=8$. Let $T$ be a Sylow $t$-subgroup of $F$. Then $T$ is a subgroup of $G$, and the vectors of $V$ fixed by $T$ form a two dimensional subspace of $V$. We can assume that $T \leqq G_{U}$. Since $t$ does not divide $\left|G L_{2}(q)\right|, T$ is contained in $L$. Then $\tilde{T} \simeq T$ is a nontrivial $t$-subgroup of $\tilde{L}$. Consequently, $\tilde{G}_{U} / \tilde{L}$ is metacyclic by 1.6 . But $\tilde{G}_{U} / \tilde{L}$ is isomorphic to $\bar{G}_{U} / \bar{K}$. Since $\bar{G}_{U} \simeq G L_{2}(q)$, either $\bar{K}$ contains $\bar{H}$ or else $q=2$ or 3 , where $\bar{H} \simeq S L_{2}(q)$.

Suppose $\bar{K} \geqq \bar{H}$. If $J \neq 1$, then $G_{U}$ contains an element $1 \neq x$ of the form

$$
\left|\begin{array}{ccc}
1 & 0 & 0 \\
0 & 1 & \\
\phi_{1} & \phi_{2} & I
\end{array}\right|
$$

where $\phi_{1}$ and $\phi_{2}$ are column vectors of length $n-2$. Since $\bar{K} \geqq \bar{H}, K$ contains an element $y$ of the form

$$
\left|\begin{array}{ccc}
1 & 0 & 0 \\
a & 1 & \\
\theta_{1} & \theta_{2} & I
\end{array}\right|
$$


where $a \neq 0$ and $\theta_{1}$ and $\theta_{2}$ are column vectors of length $n-2$. Now we calculate the commutator $[x, y]$.

$$
[x, y]=\left|\begin{array}{ccc}
1 & 0 & 0 \\
0 & 1 & 0 \\
-a \phi_{2} & 0 & I
\end{array}\right|
$$

Since $a \neq 0$, either $\phi_{2}=0$ or else $[x, y]$ is a transvection. But $\phi_{2}=0$ implies that $\phi_{1} \neq 0$ and $x$ is a transvection. Thus, $G$ contains a transvection, whence 1.2 implies that $G=S L(V)$. This contradiction shows that $J=1$. Then $K$ is isomorphic to $\bar{K}$ and $H$ is isomorphic to $\bar{H} \simeq$ $S L_{2}(q) . H$ consists of elements of the form:

$$
\left|\begin{array}{cc}
A & 0 \\
\theta(A) & I
\end{array}\right|
$$

where $A \in S L_{2}(q)$ and $\theta(A)$ is an $n-2$ by 2 matrix. Since $J=1, \theta$ is a function. In fact a simple computation shows that $\theta(A B)=\theta(A) B+\theta(B)$. Let $\theta_{i}(A)$ denote the $i$-th row of $\theta(A)$ for $i=1,2, \ldots, n-2$. Then $\theta_{i}(A B)=$ $\theta_{i}(A) B+\theta_{i}(B)$, i.e., each $\theta_{i}$ is a derivation of $H$ on a 2 -dimensional vector space $W$ over $G F(q)$. If $q$ is odd, then 1.3 implies that there exists a vector $\phi_{i}$ in $W$ such that $\theta_{i}(A)=\phi_{i}-\phi_{i} A$. Let $\phi$ be the $n-2$ by 2 matrix with $i$-th row $\phi_{i}$. Then $\theta(A)=\phi-\phi A$. A simple change of bases yields:

$$
\left|\begin{array}{ll}
I & 0 \\
\phi & I
\end{array}\right|\left|\begin{array}{cc}
A & 0 \\
\theta(A) & I
\end{array}\right|\left|\begin{array}{cc}
I & 0 \\
\phi & I
\end{array}\right|^{-1}=\left|\begin{array}{cc}
A & 0 \\
0 & I
\end{array}\right| .
$$

But then since $\bar{H}$ contains a transvection of $U, H$ contains a transvection.

Suppose $q$ is even. Let $A$ denote the 2 by 2 matrix

$$
\left|\begin{array}{ll}
1 & 0 \\
a & 1
\end{array}\right|, \quad a \neq 0 .
$$

According to 1.4 each $\phi_{i}$ can be chosen so that $\theta_{i}(A)-\phi_{i}+\phi_{i} A$ has a zero in the second coordinate. Then the second column of $\theta(A)-\phi+\phi A$ consists entirely of zeroes. The above change of basis shows that $G$ contains a transvection. Consequently, in either case $G$ contains a transvection and so equals $S L(V)$.

The above argument shows that $\bar{K}$ does not contain $\bar{H}$, so that $q=2$ or 3. Suppose $q=3$. Since $\bar{G}_{U} / \bar{K}$ is metacyclic but $\bar{K}$ does not contain $\bar{H}, \bar{K}$ is the Sylow 2-subgroup of $\bar{H} \simeq S L_{2}$ (3). In this case $K$ contains an element $x$ of the form

$$
\left|\begin{array}{cc}
-I & 0 \\
0 & I
\end{array}\right|
$$


Let $P$ be a one dimensional subspace of $U$. If $X \leqq G_{P}$, let $\bar{X}$ denote the image of $X$ acting on $V / P=\bar{V}$. Then $\bar{x}$ is a dilatation of $\bar{V} G$ is doubly transitive on $L_{1}(V)$, and so $\bar{G}_{P}$ is transitive on $L_{1}(\bar{V}) .1 .7$ now implies that $\bar{G}_{P}$ contains $S L(\bar{V})$. It is easy to show that $G$ contains $S L(V)$ (for example, see [9]). This contradiction implies that $q \neq 3$.

The only remaining possibility is $q=2$ and $\bar{K} \neq \bar{H}$. In this case $\tilde{G}_{U} / \tilde{L} \simeq \vec{G}_{U} / \bar{K}$ has order divisible by 2 . According to $1.6,2$ divides $(n-2)\left(2^{n-2}-1\right)$, whence 2 divides $n$.

\section{Similar Results}

In this final section we give several results whose proof is similar to that of Theorem 1 .

Theorem 2. Suppose $V$ is a vector space of finite dimension $n \geqq 7$ over $G F(2)$. Suppose $G \leqq S L(V)$ acts transitively on $L_{4}(V)$. Then $G=S L(V)$.

Proof. The proof is similar to the proof of Theorem 1 with $U$ equal to a three dimensional subspace of $V$ instead of a two dimensional subspace. The proof goes through because of the simplicity of $S L_{3}(2)$ and the particular form of the derivations of $S L_{3}(2)$ on its standard module. For $n=9$ there is a slight difficulty since $2^{n-3}-1=63$ has no 2-primitive divisor. Also, for $n=7$ elements of $L_{4}(V)$ are dual to elements of $L_{3}(V)$. However, in both cases $G$ is transitive on $L_{3}(V)$ and the dimension of $V$ is odd, whence Theorem 1 implies that $G$ equals $S L(V)$.

Theorem 3. Let $V$ be a vector space of finite dimension $n \geqq 4$ over a finite field $G F(q)$. Suppose $G \leqq G L(V)$ acts on $L_{2}(V)$ as a rank three permutation group. Then $G \geqq S L(V)$.

Proof. Since $G$ is transitive on $L_{3}(V)$, the result follows from Theorem 1 if $q \neq 2$ and $n \geqq 6$. Since $G$ has subdegrees $1, q(q+1)\left(q^{n-2}-1\right)$ / $(q-1)$, and $q^{4}\left(q^{n-2}-1\right)\left(q^{n-3}-1\right) /(q-1)\left(q^{2}-1\right)$, for $n>5$ the order of $G$ is divisible by $q$-primitive divisors of $q^{n-2}-1$ and $q^{n-3}-1$. Hence, for $q=2$ and $n \geqq 7$ the proof goes through as in Theorem 2. By $1.1 \mathrm{G}$ acts doubly transitively on $L_{1}(V)$. Thus for $n \leqq 6$ the result follows from work of Wagner [9] and unpublished work of Higman. The only group $G$ which acts doubly transitively on $L_{1}(V)$ for $n<7$ and does not contain $S L(V)$ is a subgroup $H$ of $G L_{4}(2)$ which is isomorphic to $A_{7}$. However, its action on $L_{2}(V)$ has rank greater than 3 .

We give one final application of 1.5 and 1.6 to a problem of Higman and McLaughlin [5]. Let $f$ be a nondegenerate alternate or hermitian bilinear form on a vector space $V$ of finite dimension $n \geqq 4$ over $G F(q)$, where $q=q_{0}^{2}$ if $f$ is hermitian and $q=q_{0}$ if $f$ is alternate. Let $A$ denote the subset of $L_{1}(V)$ consisting of those one dimensional subspaces $\langle v\rangle$ 
such that $f(v, v)=0$. Let $G U(f)$ denote the group of all transformations in $G L(V)$ which preserve $f$, and let $S U(f)=G U(f) \cap S L(V)$. $S U(f)$ acts on $A$ as a rank 3 permutation group. The problem is to determine all groups $H \leqq G U(f)$ which act on $A$ as a rank 3 permutation group. Higman and McLaughlin showed that for $n \leqq 8$, any such group contains $S U(f)$ with the possible exception of $q$ even and $f$ alternate. It is possible to remove this restriction on $n$ by applying 1.5 and 1.6. The maximal subgroups of $\mathrm{Sp}_{4}(q), q$ even, have been determined by Flesner [3]. The only proper subgroup of $\mathrm{Sp}_{4}(q)$ which acts on $A$ as a rank 3 permutation group is the commutator subgroup of $\mathrm{Sp}_{4}(2)$, a group isomorphic to $A_{6}$. Thus, for $n=4$ all subgroups $H \leqq G U(f)$ which act on $A$ as a rank 3 permutation group have been determined. Consequently, we assume $n \geqq 5$.

Suppose $G \leqq G U(f)$ acts on $A$ as a rank 3 permutation group but does not contain $S U(f)$. We show that $q=2$ and $f$ is alternate. For $P \in A$ let $B(P)$ denote the set of points $Q \in A$ which are distinct from $P$ and lie in $P^{\perp}$. Let $C(P)$ denote the subset of $A$ consisting of those points which do not lie in $P^{\perp}$. Then $\{P\}, B(P)$, and $C(P)$ are the three orbits of $G_{P}$ acting on $A$. Let $U$ be a two dimensional subspace of $V$ such that the restriction of $f$ to $U$ is nondegenerate. Let $P$ be a point in $L_{1}(U) \cap A$. Let $\bar{G}_{U}$ denote the image of $G_{U}$ acting on $U$. Since $B(P) \cap L_{1}(U)=\emptyset$, $\bar{G}_{U}$ acts doubly transitively on the $q_{0}+1$ points of $A \cap L_{1}(U)$. Hence $\bar{G}_{U}$ contains a subgroup isomorphic to $S L_{2}\left(q_{0}\right)$.

Let $L$ denote the subgroup of $G_{U}$ which acts trivially on $U$. $L$ is a normal subgroup of $G_{U}$, and $G_{U} / L \simeq \bar{G}_{U}$. According to Theorem 5 of [5], $G_{U}$ acts faithfully on $U^{\perp}$. Thus, $G_{U}$ is a linear group which has a homomorphic image containing a subgroup isomorphic to $S L_{2}\left(q_{0}\right)$.

Let $K(n)=\left(q^{n}-1\right) /(q-1)$ if $f$ is alternate and $\left(q_{0}^{n}-(-1)^{n}\right)\left(q_{0}^{n-1}-(-1)^{n-1}\right) /(q-1)$ if $f$ is hermitian. Then $|A|=K(n)$, $|B(P)|=q K(n-2)$, and $|C(P)|=q_{0} q^{n-2}$. Suppose that $f$ is alternate. Then $\left|G_{P}\right|$ is divisible by $|B(P)|=q\left(q^{n-2}-1\right) /(q-1)$. Let $t$ be a $q$-primitive prime divisor of $q^{n-2}-1$. Since $n-2>2,1.5$ implies that such a divisor exists except for $q=2$ and $n-2=6$. Let $T$ be a Sylow $t$-subgroup of $G_{P}$. Since $(t, q)=1, T$ fixes some point $Q \in C(P)$, whence $T$ fixes the two dimensional subspace spanned by $P$ and $Q$. We can assume that $T$ fixes $U$. Since $n-2>2, t$ does not divide $\left|G L_{2}(q)\right|$, and so $T$ is contained in $L$. 1.6 now implies that $G_{U} / L$ is metacyclic. Since $G_{U} / L$ is isomorphic to $S L_{2}(q), q$ must equal 2.

Suppose that $f$ is hermitian and $n$ is odd. In this case $\left|G_{p}\right|$ is divisible by $q_{0}^{n-2}+1$. Let $t$ be a $q$-primitive prime divisor of $q^{n-2}-1=$ $\left(q_{0}^{n-2}-1\right)\left(q_{0}^{n-2}+1\right)$. Such a divisor exists by 1.5 since $n-2>2$ and $q$ is a square. The prime $t$ divides $q_{0}^{n-2}+1$ and so also divides $\left|G_{P}\right|$. Let $T$ be a Sylow $t$-subgroup of $G_{P}$. $T$ fixes some point $Q \in C(P)$, and we can 
assume that $L$ contains $T$. 1.6 now implies that $G_{U} / L$ is a metacyclic group of order dividing $(n-2)\left(q^{n-2}-1\right)$. Since $G_{U} / L$ contains a subgroup isomorphic to $S L_{2}\left(q_{0}\right)$ and $n$ is odd, this is not possible.

Finally suppose that $f$ is hermitian and $n$ is even. Then $\left|G_{P}\right|$ is divisible by $q_{0}^{n-3}+1$. Let $t$ be a $q$-primitive divisor of $q^{n-3}-1$, and let $T$ be a Sylow $t$-subgroup of $G_{p}$. We can assume $T \leqq G_{V}$. Since $n \geqq 6, t$ does not divide $\left|G L_{2}(q)\right|$. Consequently, $T$ is contained in $L$. The Frattini argument implies that $G_{V} / L$ is the homomorphic image of a subgroup of the normalizer of $T$ in $G U\left(U^{\perp}\right)$. But $T$ acts on $U^{\perp}$ by fixing a one dimensional subspace and a complementary hyperplane on which it acts as a subgroup of a Singer subgroup. Hence, the normalizer of $T$ in $G L\left(U^{\perp}\right)$ is the direct product of a cyclic group of order $q-1$ and a metacyclic group of order $(n-3)\left(q^{n-3}-1\right)$. In particular this group is solvable. Since $G_{U} / L$ contains a group isomorphic to $S L_{2}\left(q_{0}\right), q_{0}$ must equal 2 or 3. But $q_{0}=2$ is impossible since then $(q-1)(n-3)\left(q^{n-3}-1\right)$ is odd. Since $S L_{2}(3)$ has no normal cyclic subgroup with a metacyclic factor group, $q_{0}=3$ is also impossible. The proof of the following theorem is now complete.

Theorem 4. If $G \leqq G U(f)$ acts on $A$ as a rank 3 permutation group, then $G$ contains $S U(f)$ with the possible exception of $q=2$ and $f$ alternate.

\title{
References
}

1. Artin, E.: The orders of the linear groups. Commun. Pure Appl. Math. 8, 355-365 (1955).

2. Birkhoff, G.D., Vandiver, W.S.: On the integral divisors of $a^{n}-b^{n}$. Ann. of Math. (ser. 2) 5, 173-180 (1904).

3. Flesner, D.: Maximal subgroups of the four-dimensional symplectic groups in characteristic two. Thesis, University of Michigan, 1971.

4. Hering, $C$.: Zweifach transitive Permutationsgruppen, in denen 2 die maximale Anzahl von Fixpunkten von Involutionen ist. Math. Z. 104, 150-174 (1968).

5. Higman, D.G., McLaughlin, J.E.: Rank 3 subgroups of finite symplectic and unitary groups. J. reine angew. Math. 218, 174-189 (1965).

6. Kantor, W.: Line-transitive collineation groups of finite projective spaces. Preprint.

7. McLaughlin, J.E.: Some groups generated by transvections. Arch. der Math. 18, 364-368 (1967)

8. Piper, F.C.: Collineation groups containing homologies. J. Algebra 6, 256-269 (1967).

9. Wagner, A.: On collineation groups of projective spaces I. Math. Z. 76, 411-426 (1961).

\author{
Dr. Davia Perin \\ Department of Mathematics \\ University of Michigan \\ Ann Arbor, Mich. 48104 \\ USA
}

(Received June 14,1971) 